\title{
Acylated and unacylated ghrelin inhibit apoptosis in myoblasts cocultured with colon carcinoma cells
}

\author{
XIANLIANG ZENG $^{1}$, SIZENG CHEN $^{1}$, YANFENG LIN $^{2}$ and ZHAO KE $^{1}$ \\ ${ }^{1}$ Department of Gastrointestinal Surgery, The First Affiliated Hospital of Fujian Medical University; \\ ${ }^{2}$ Department of Laboratory Medicine, Medical Technology and Engineering College, \\ Fujian Medical University, Fuzhou, Fujian 350004, P.R. China
}

Received July 24, 2017; Accepted December 20, 2017

DOI: $10.3892 / o r .2018 .6213$

\begin{abstract}
Cancer cachexia is a life-threatening syndrome associated with myofiber damage. Tumor factors impair muscle regeneration by promoting myoblast apoptosis. Ghrelin is a multifunctional hormone with an anti-apoptotic effect, but its mechanism of action is not fully understood. In the present study, we investigated whether the coculturing of $\mathrm{C} 2 \mathrm{C} 12$ myoblasts with CT26 colon carcinoma cells may induce myoblast apoptosis, and whether acylated ghrelin (AG) and unacylated ghrelin (UnAG) may exert anti-apoptotic effects. We found that the coculture induced myoblast apoptosis and increased tumor necrosis factor (TNF)- $\alpha$ concentrations in the culture medium. Moreover, the coculture increased c-Jun N-terminal kinase (JNK) activity, suppressed Akt activity, increased the mitochondrial $\mathrm{Bax} / \mathrm{Bcl}-2$ ratio, impaired mitochondrial membrane potential $\left(\Delta \psi_{\mathrm{m}}\right)$, increased the cytosolic cytochrome $c$ levels, and activated the caspase-3/poly (ADP-ribose) polymerase (PARP) cascade in myoblasts. We also found that either AG or UnAG inhibited these changes. The present study describes a novel in vitro model that can be employed to investigate cancer-induced myoblast apoptosis, and our findings suggest a possible use for AG and UnAG in treating cancer cachexia.
\end{abstract}

Correspondence to: Professor Sizeng Chen, Department of Gastrointestinal Surgery, The First Affiliated Hospital of Fujian Medical University, 20 Chazhong Road, Fuzhou, Fujian 350004, P.R. China

E-mail: chensz04871@hotmail.com

Abbreviations: AG, acylated ghrelin; UnAG, unacylated ghrelin; TNF- $\alpha$, tumor necrosis factor- $\alpha$; JNK, c-Jun N-terminal kinase; CYTC, cytochrome $c$; PARP, poly(ADP-ribose) polymerase; MOMP, mitochondrial outer membrane permeabilization; MOM, mitochondrial outer membrane; MAPK, mitogen-activated protein kinase; PI3K, phosphatidylinositol-3-kinase; Akt, protein kinase B; IL-1 $\beta$, interleukin- $1 \beta$; IFN- $\gamma$, interferon- $\gamma$

Key words: cancer cachexia, apoptosis, ghrelin, coculture, myoblast

\section{Introduction}

Cancer cachexia is a multifactorial metabolic syndrome characterized by muscle wasting (with or without fat wasting) and systemic inflammation (1). It occurs in nearly $85 \%$ of terminal cancer patients, and is responsible for $\sim 20 \%$ of all cancer-related deaths (2). Muscle atrophy is the major physiological effect of cancer cachexia. It is induced by multiple mechanisms, including an imbalance between muscle protein synthesis and degradation $(3,4)$, myocyte apoptosis (5-7), and muscle regeneration dysfunction (8). Muscle regeneration by satellite cells is the main means of repairing muscle damage. Muscle damage, particularly to the sarcolemma, is an important feature of cancer cachexic muscle atrophy. In animals and humans with cancer cachexic, various tumor factors impair not only the sarcolemma, but also the ability to regenerate muscle tissue.

Satellite cells are undifferentiated mononuclear myogenic cells (9). In resting adult muscles, satellite cells are quiescent in a reversible G0 state. When muscle fibers are damaged, satellite cells become activated and proliferate to produce muscle precursor cells, commonly referred to as myoblasts (10). The myoblasts then fuse into existing myofibers in need of repair (11). Tumor factors can inhibit myoblasts from fusing into myofibers and thereby induce myoblast apoptosis, which impairs muscle regeneration $(8,12)$.

The most common mechanism of apoptosis in normal and diseased tissue involves the mitochondrial pathway (13). Apoptotic stimuli converge at mitochondria and cause mitochondrial outer membrane permeabilization (MOMP) (14). The Bcl-2 family of proteins plays a critical role in apoptosis by regulating mitochondrial integrity (15). The Bcl-2 protein family contains both pro- and anti-apoptotic members, and the Bax protein is a pro-apoptotic member. During apoptosis, cytosolic Bax translocates to pores in the mitochondrial outer membrane (MOM), where it impairs mitochondrial integrity, induces the loss of mitochondrial membrane potential $\left(\Delta \psi_{\mathrm{m}}\right)$, and causes the release of cytochrome $c$ (CYTC) into the cytosol. The released CYTC then triggers the caspase-3/poly (ADP-ribose) polymerase (PARP) proteolytic cascade, which induces apoptosis. Bid is another pro-apoptotic member of the Bc1-2 protein family. JNK activates caspase- 8 and thereby induces Bid cleavage (16). The resulting truncated tBid protein 
then binds to Bax, and initiates the activation of Bax (15). As an anti-apoptotic member of the Bc1-2 family, the Bc1-2 protein binds with Bax on the MOM, and then inactivates Bax to protect mitochondrial integrity.

Ghrelin is a multifunctional circulating hormone that consists of 28 amino acids and exists in two different forms: acylated ghrelin (AG) and unacylated ghrelin (UnAG), respectively. Both forms originate from the same precursor, and the only structural difference between them is an octanoylated Ser3 found in AG. Both AG and UnAG are predominantly synthesized in stomach cells and then secreted into blood serum $(17,18)$. Ghrelin receptors are widely expressed in the central nervous system, intestines, pancreas, liver, adipose tissue, skeletal and cardiac muscle, and play important roles in numerous biological functions, including appetite regulation, gastric motility, pancreatic, cardiovascular and immune function, and muscle metabolism in both humans and animals (18-20). Both AG and UnAG can act directly on myoblasts to promote their differentiation and fusion, although the identity of their receptor(s) remains unknown (21). Accumulating evidence suggests that $A G$ and $U n A G$ inhibit apoptosis via the mitogen-activated protein kinase (MAPK) and phosphatidylinositol-3-kinase (PI3K)/Akt pathways (22-24). Moreover, AG/UnAG also regulate Bcl-2 family proteins and inhibit apoptosis by preventing mitochondrial dysfunction $(25,26)$.

It has never been investigated whether AG or UnAG may inhibit myoblast apoptosis induced by tumor factors. In the present study, we used a Transwell-plate system to develop a novel myoblast-carcinoma cell coculture model. This model allows myoblasts and carcinoma cells to grow in the same culture medium and establish intercellular communications without the need for cell-to-cell contact. We then examined whether this type of culture environment induced myoblast apoptosis. We also investigated whether AG or UnAG inhibited myoblast apoptosis, and if so, the possible mechanisms involved.

\section{Materials and methods}

Cell culture. Mouse C2C12 myoblasts and CT26 colon carcinoma cells were obtained from the American Type Culture Collection (ATCC; Manassas, VA, USA) and maintained, respectively, in Dulbecco's modified Eagle's medium (DMEM) (Invitrogen, Carlsbad, CA, USA) that was supplemented with $10 \%$ fetal bovine serum (FBS) (Gibco, Auckland, New Zealand) and $1 \%$ penicillin-streptomycin (Invitrogen). The cells were cultured in an atmosphere of $5 \% \mathrm{CO}_{2}$ at $37^{\circ} \mathrm{C}$.

To establish the coculture model, $\mathrm{C} 2 \mathrm{C} 12$ myoblasts were seeded into the lower wells of a 6-well Transwell clear plate (3450; Corning, Corning, NY, USA) at a density of 20,000 cells $/ \mathrm{cm}^{2}$; CT26 cells were seeded $\left(20,000\right.$ cells $\left./ \mathrm{cm}^{2}\right)$ into the upper inserts $(0.4-\mu \mathrm{m}$ pore polyester membrane) of another 6-well plate (\#3516; Corning). After $24 \mathrm{~h}$ of culture, the upper inserts were placed into the lower wells that contained myoblasts. The base of each insert contained a membrane with $0.4-\mu \mathrm{m}$ pores that allowed the movement of secreted factors, and thus permitted paracrine interactions to occur between the two different cell types. Next, the medium in both the lower wells and upper inserts was changed to fresh medium with or without AG/UnAG (1465/2951, $100 \mathrm{nM}$; Tocris Bioscience (Ellisville, MO, USA), and the cells were cocultured for $24 \mathrm{~h}$. After $24 \mathrm{~h}$ of coculture, the medium was collected for analysis by ELISA, and the myoblasts were harvested for analysis by RT-qPCR, western blotting, flow cytometry or the 5,5',6,6'-tetrachloro-1,1',3,3'-tetraethylbenzimidazole-carbocyanine iodide (JC-1) staining assay.

The coculture combinations consisted of sham myoblasts (without CT26 cells in the insert) and without ghrelin (NC group); myoblasts with CT26 cells but without ghrelin (CO group); myoblasts with CT26 cells and acylated ghrelin (AG group); myoblasts with CT26 cells and unacylated ghrelin (UnAG group).

Flow cytometry analysis. Both adherent and floating myoblasts were collected and washed twice with PBS. An Annexin V/PI Staining kit (C1062; Beyotime, Shanghai, China) was used according to the manufacturer's instructions to quantitatively assess the apoptosis status of the myoblasts. A Mitochondrial Membrane Potential Assay kit with JC-1 (C2006; Beyotime) was used according to the manufacturer's instructions to assess the mitochondrial membrane potential of myoblasts. Fluorescence intensity was detected with an Accuri C6 flow cytometer (BD Biosciences, San Diego, CA, USA). A minimum of $1 \times 10^{5}$ cells were recorded in each sample, and results were analyzed using FlowJo software (version 7.6.2, FlowJo; LLC, Ashland, OR, USA).

JC-1 staining assay. Myoblasts were washed twice with PBS, and then incubated with JC-1 dye (C2006; Beyotime) according to the manufacturer's instructions. Images of stained cells were acquired with an Olympus IX71 fluorescence microscope (Olympus Corporation, Tokyo, Japan). JC-1 dye can exist in two different states: aggregates and monomers. When excited at $488 \mathrm{~nm}$, monomers emit at $530 \mathrm{~nm}$ (green) and aggregates emit at $590 \mathrm{~nm}$ (red). Red emissions signify healthy mitochondria, as healthy mitochondria are polarized, and the JC-1 taken up by such mitochondria forms aggregates. JC-1 does not accumulate in depolarized mitochondria, but rather remains in the cytoplasm as monomers.

Western blot analysis. Myoblasts were washed twice with ice-cold PBS, and then scraped into a $4^{\circ} \mathrm{C}$ cell lysis buffer (\#9803; Cell Signaling Technology, Danvers, MA, USA) that was supplemented with a protease inhibitor cocktail (05892970001; Roche Diagnostics GmbH, Mannheim, Germany). Protein concentrations were measured using a BCA kit (BCA1; Sigma-Aldrich, St. Louis, MO, USA). Mitochondrial proteins were isolated using a Mitochondria Isolation kit for Cultured Cells (ab110170; Abcam, Cambridge, $\mathrm{UK})$. Aliquots of total protein (20 $\mu \mathrm{g} / \mathrm{lane})$ were separated by electrophoresis on a 4-20\% SDS-PAGE gel, and the separated proteins bands were transferred onto PVDF membranes. The membranes were blocked with $5 \%$ skim milk, and then incubated overnight at $4^{\circ} \mathrm{C}$ with a primary antibody; after which, they were incubated with a HRP-conjugated secondary antibody (ab97051, 1:2,000; Abcam). The immunostained proteins were visualized with enhanced chemiluminescence reagents (GE2301; GenView Scientific, Arcade, NY, USA). Images of the membranes were recorded with a ChemiDoc 
A

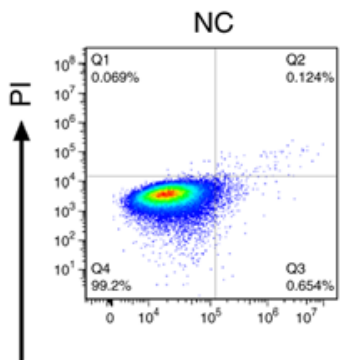

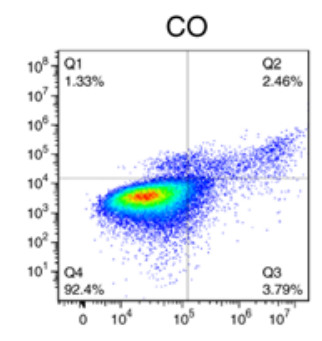
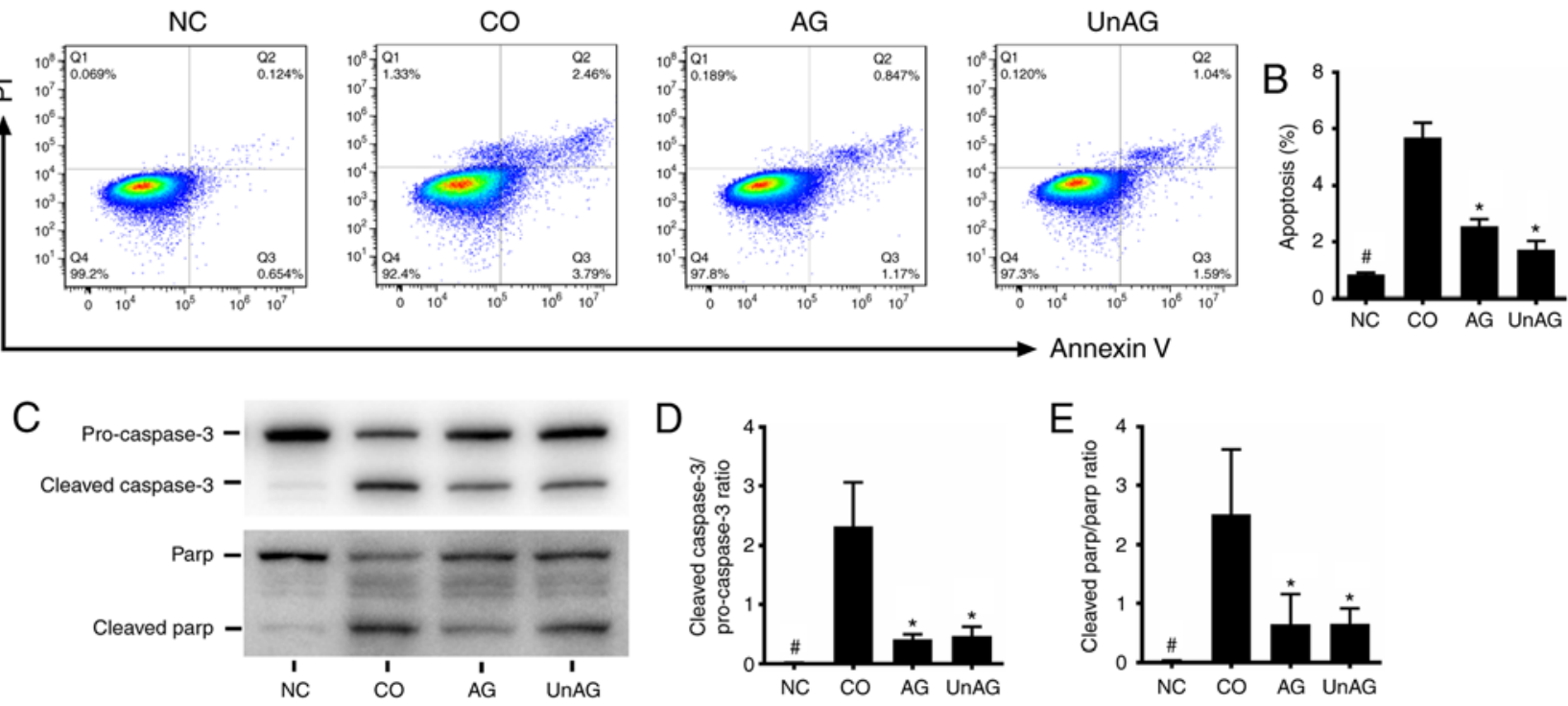

Annexin V

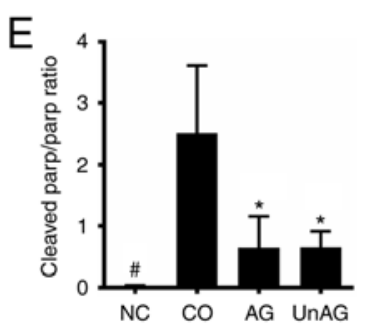

Figure 1. Effect of AG and UnAG on coculture-induced apoptosis in myoblasts. (A) Twenty-four hours after coculture, apoptosis of myoblasts was detected by flow cytometry. The signals from apoptotic myoblasts are localized in the Q2 and Q3 quadrants of the resulting pseudocolor graph. (B) Statistical graph of apoptosis in the different groups. Significant differences were detected between $\mathrm{CO}$ and $\mathrm{NC}$ groups $\left({ }^{\#} \mathrm{P}<0.001\right)$, between $\mathrm{CO}$ and $\mathrm{AG} / \mathrm{Un} \mathrm{AG}$ groups ( $\mathrm{P}<0.001)$, by one-way ANOVA followed by Tukey's test, $\mathrm{F}=126.284, \mathrm{P}<0.0001$. (C) Western blotting of cleaved caspase-3, pro-caspase-3, cleaved-PARP, PARP and GAPDH in myoblasts. (D) Quantification of cleaved caspase-3/pro-caspase-3 ratio. Significant differences were detected between CO and NC groups $\left({ }^{\#} \mathrm{P}<0.001\right)$, between $\mathrm{CO}$ and $\mathrm{AG} / \mathrm{UnAG}$ groups $\left({ }^{*} \mathrm{P}=0.001, \mathrm{P}=0.002\right.$; respectively), by one-way ANOVA followed by Tukey's test, $\mathrm{F}=21.587, \mathrm{P}<0.0001$. (E) Quantification of cleaved-PARP/PARP ratio. Significant differences were detected between CO and NC groups ( $\left.{ }^{\mathrm{P}}=0.005\right)$, between $\mathrm{CO}$ and AG/UnAG groups $\left({ }^{*} \mathrm{P}=0.025, \mathrm{P}=0.027\right.$; respectively), by one-way ANOVA followed by Tukey's test, $\mathrm{F}=8.927, \mathrm{P}=0.0006$. Data are represented as mean $\pm \mathrm{SD}$. The coculture combinations consisted of sham myoblasts (without CT26 cells in the insert) and without ghrelin (NC group); myoblasts with CT26 cells but without ghrelin (CO group); myoblasts with CT26 cells and acylated ghrelin (AG group); myoblasts with CT26 cells and unacylated ghrelin (UnAG group).

XRS+ System, and analyzed using Quantity One software (version 4.6.6) (both from Bio-Rad, Hercules, CA, USA). The following antibodies were used as primary antibodies: Abcam: anti-caspase-3 (ab184787); anti-PARP (ab191217); anti-Bax (ab32503); anti-Bcl-2 (ab182858); anti-Bcl-2 (phospho-S70, ab138406); anti-Bid (ab63541); anti-Bid cleavage site (ab10640); anti-cytochrome $c$ (ab133504); anti-COX IV (ab202554); anti-p38 (ab170099); anti-p38 (phospho-T180+ Y182, ab195049); anti-JNK1 + JNK2 + JNK3 (ab179461); anti-JNK1 + JNK2 + JNK3 (phospho-Y185 + Y185 + Y223, ab76572); anti-ERK1 + ERK2 (ab184699); anti-Erk1 (phosphoT202 + Y204) + Erk2 (phospho-T185 + Y187) (ab76299); anti-Akt (ab179463); anti-Akt (phospho-S473, ab81283); anti-GSK3 $\beta$ (ab32391); anti-GSK3 $\beta$ (phospho-S9, ab131097); anti-GAPDH (ab181602).

Real-time quantitative RT-PCR analysis. Total RNA was extracted from myoblasts with TRIzol reagent (15596026; Invitrogen) in accordance with the manufacturer's instructions. Reverse transcription was performed with SuperScript II reverse transcriptase (18064022; Invitrogen). The resulting cDNA for specific transcripts was used for real-time quantitative PCR (RT-qPCR) performed with PowerUp SYBR-Green Master Mix (A25742; Life Technologies, Carlsbad, CA, USA) and a 7500 Real-Time PCR System (Applied Biosystems, Foster City, CA, USA). Gene expression data was normalized to that of a housekeeper gene (GAPDH). Relative gene expression levels were obtained using the $2^{-\Delta \Delta \mathrm{Ct}}$ method. The following RT-qPCR primer sequences were used: Bcl-2, 5'-GTGGTGGAGGAACTCTTCAG-3' and 5'-GTTCCACAA AGGCATCCCAG-3'; GAPDH, 5'-ATGACAATGAATACG

\section{GCTACAGCAA-3' and 5'-GCAGCGAACTTTATTGATGGT} ATT-3'.

ELISA. A Mouse TNF alpha ELISA kit (ab46105), Mouse IL-1 beta ELISA kit (ab100704) (both from Abcam), and Mouse IFN-gamma Quantikine ELISA kit (MIF00; R\&D Systems; Minneapolis, MN, USA) were used to measure TNF- $\alpha$, IL- $1 \beta$ and IFN- $\gamma$ concentrations, respectively, in samples of cell culture medium according to the manufacturer's instructions. The assay plates were read using a SpectraMax M5 microplate reader (Molecular Devices, Sunnyvale, CA, USA).

Statistical analysis. Each experiment was repeated at least three times, and all data were analyzed using IBM SPSS Statistics for Windows, version 19.0 (IBM Corp., Armonk, NY, USA). Results are shown as the mean \pm SD. Statistical comparisons between groups were analyzed using one-way ANOVA followed by the Tukey's test when equal variances were assumed. When equal variances were not assumed, Dunnett's T3 test was applied. Two-sided P-values $<0.05$ were considered statistically significant.

\section{Results}

$A G$ and UnAG attenuate coculture-induced apoptosis in myoblasts. Fig. 1A and B show results of the flow cytometric assays used to assess the apoptotic status of myoblasts. The coculture significantly increased the numbers of myoblasts undergoing apoptosis $(\mathrm{P}<0.001)$, and both $\mathrm{AG}$ and UnAG attenuated those increases $(\mathrm{P}<0.001)$. Moreover, as shown in 
A

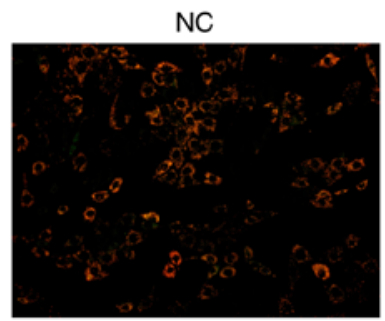

B

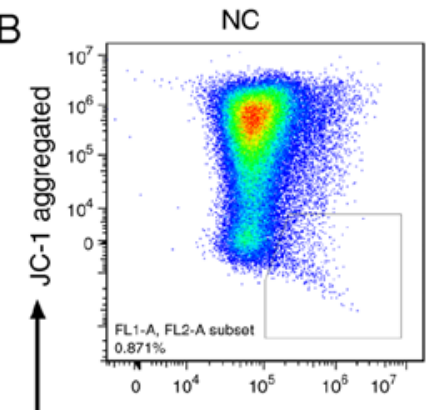

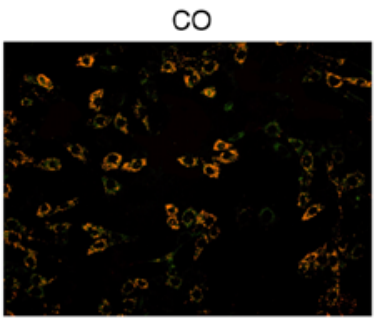

CO

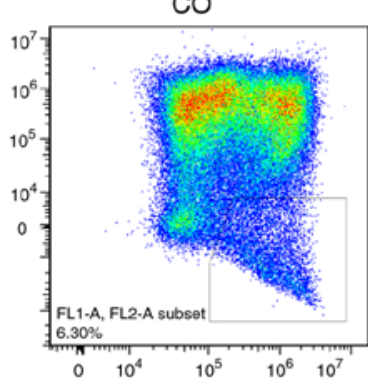

AG

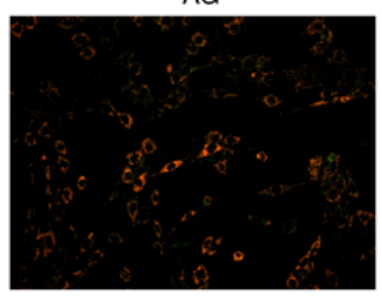

AG

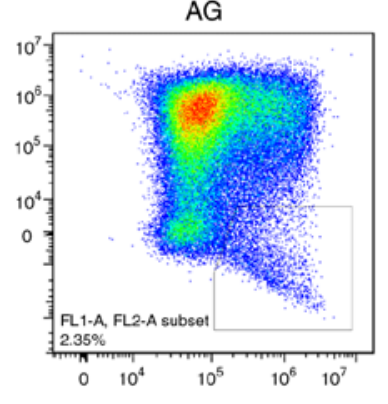

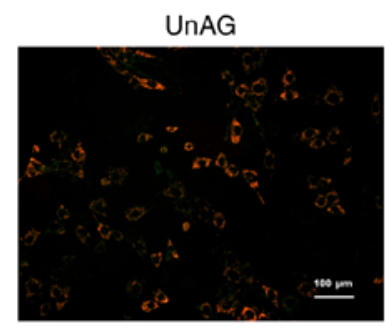

UnAG

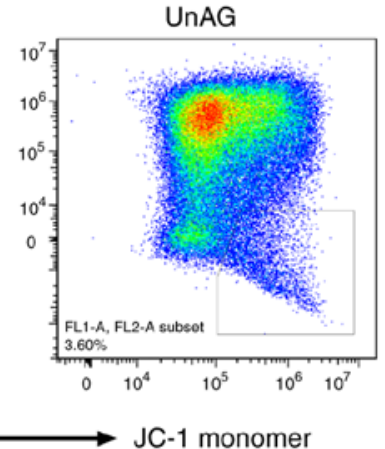

F

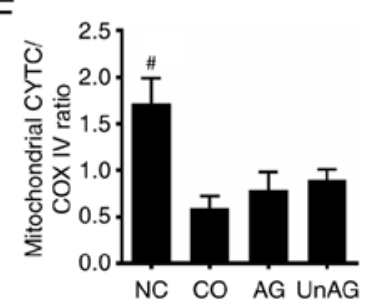

Figure 2. Effect of AG and UnAG on coculture-induced mitochondrial dysfunction in myoblasts. (A) Detection of mitochondrial membrane potential by JC-1 staining (x100). Scale bar represents $100 \mu \mathrm{m}$. (B) Flow cytometry analysis of JC-1-stained myoblasts. (C) Statistical graph of JC-1 monomer in the different groups $(\mathrm{n}=4)$. Significant differences were detected between $\mathrm{CO}$ and $\mathrm{NC}$ groups $\left({ }^{\#} \mathrm{P}=0.001\right)$, between $\mathrm{CO}$ and $\mathrm{AG} / \mathrm{UnAG}$ groups $\left({ }^{*} \mathrm{P}=0.009\right.$, $\mathrm{P}=0.002$; respectively), by one-way ANOVA followed by Dunnett's T3 test, F=149.332, $\mathrm{P}<0.0001$. (D) Western blotting of cytosolic cytochrome $\mathrm{c}$ (CYTC), mitochondrial CYTC, GAPDH and COX IV in myoblasts. (E) Quantification of cytosolic CYTC was normalized to GAPDH. Significant differences were detected between $\mathrm{CO}$ and NC groups ( $\left.{ }^{\#} \mathrm{P}<0.001\right)$, between $\mathrm{CO}$ and $\mathrm{AG} / \mathrm{UnAG}$ groups $\left({ }^{*} \mathrm{P}<0.001\right)$, by one-way ANOVA followed by Tukey's test, F=286.821, $\mathrm{P}<0.001$. (F) Quantification of mitochondrial CYTC was normalized to COX IV. Significant differences were detected between CO and NC groups $\left({ }^{\sharp} \mathrm{P}<0.001\right)$, by one-way ANOVA followed by Tukey's test, $\mathrm{F}=20.972, \mathrm{P}<0.001$. Data are represented as mean $\pm \mathrm{SD}$. CYTC, cytochrome c. The coculture combinations consisted of sham myoblasts (without CT26 cells in the insert) and without ghrelin (NC group); myoblasts with CT26 cells but without ghrelin (CO group); myoblasts with CT26 cells and acylated ghrelin (AG group); myoblasts with CT26 cells and unacylated ghrelin (UnAG group).

Fig. 1C-E, a western blot analysis revealed that the coculture was associated with an increased level of cleaved caspase-3 protein and a decreased level of pro-caspase- 3 protein, which increased the cleaved caspase-3/pro-caspase-3 ratio in myoblasts. Moreover, similar changes were observed in the cleaved-PARP/PARP ratio, and AG and UnAG also attenuated these changes.

$A G$ and UnAG ameliorate the coculture-induced mitochondrial dysfunction in myoblasts. The JC-1 stain flow cytometric assay was used to assess the $\Delta \psi_{\mathrm{m}}$ of mitochondria in the myoblasts. As shown in Fig. 2A-C, the coculture significantly decreased the membrane potential of mitochondria in the myoblasts $(\mathrm{P}<0.01)$, and both AG and UnAG ameliorated these changes $(\mathrm{P}<0.01)$. Moreover, western blot analysis revealed that coculture-induced impairment of mitochondria resulted in the release of CYTC from the mitochondria into the cytosol, and both AG and UnAG attenuated the effect (Fig. 2D-F).

Action of Bcl-2 family proteins in myoblasts. We performed western blotting and RT-qPCR assays to assess the role of Bcl-2 family proteins. As shown in Fig. 3A, B, F and I, the coculture decreased the levels of Bcl-2 protein in mitochondria, and both AG and UnAG ameliorated these changes. However, the levels of Bc1-2 mRNA and cytosolic protein were not affected by either coculture or AG/UnAG administration. Regarding Bax, the coculture decreased its cytosolic protein levels and increased its mitochondrial protein levels (Fig. 3A, $\mathrm{C}$ and $\mathrm{G}$ ), and thus increased the $\mathrm{Bax} / \mathrm{Bcl}-2$ ratio in mitochondria Fig. $3 \mathrm{H}$ ). Moreover, the coculture also increased the levels of tBid protein and reduced the levels of Bid protein in the cytosol (Fig. 3A, D and E), and both AG and UnAG attenuated those changes.

MAPK pathway activity in myoblasts. We performed western blot assays to assess MAPK pathway activity. As shown in Fig. 4A-C, the coculture significantly increased the levels of $\mathrm{p}$-JNK and JNK proteins in the myoblasts $(\mathrm{P}<0.01)$, and both $\mathrm{AG}$ and UnAG ameliorated these changes. Moreover, the $\mathrm{p}-\mathrm{JNK} / \mathrm{JNK}$ ratio was also increased by coculture, and that increase was ameliorated by UnAG (Fig. 4D). No significant difference in the levels of p-P38, P38, p-ERK and ERK proteins was observed among the four different groups (Fig. 4E and F). 

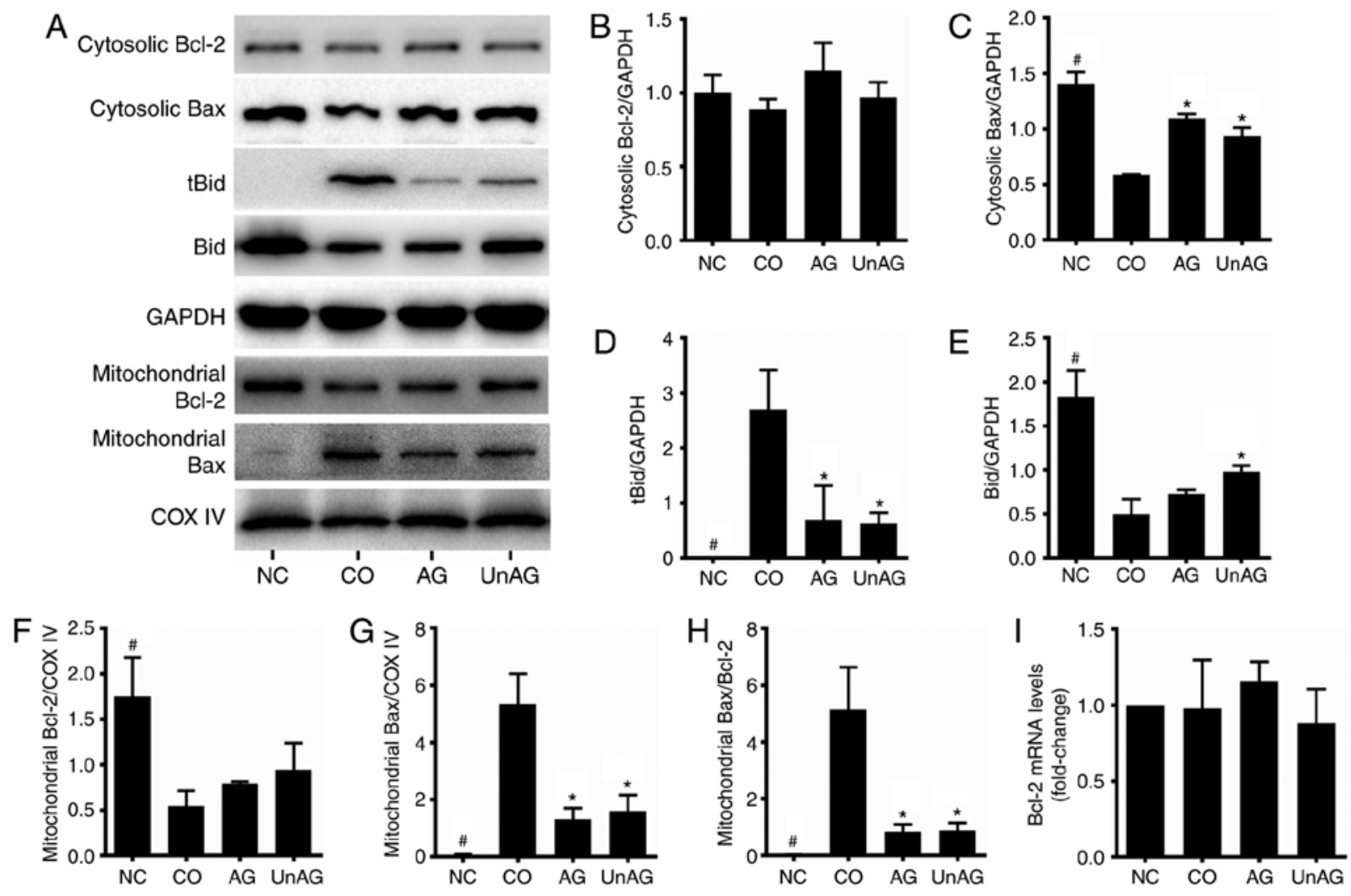

Figure 3. Regulation of Bcl-2 family proteins by coculture and AG/UnAG administration in myoblasts. (A) Western blotting of protein levels. (B) Quantification of cytosolic Bcl-2 was normalized to GAPDH. No significant differences were detected between 4 groups, by one-way ANOVA followed by Tukey's test. (C) Quantification of cytosolic Bax was normalized to GAPDH. Significant differences were detected between CO and NC groups ( ${ }^{\#}<0.001$ ), between CO and AG/UnAG groups ( $\mathrm{P}<0.001, \mathrm{P}=0.01$; respectively), by one-way ANOVA followed by Tukey's test, $\mathrm{F}=71.961, \mathrm{P}<0.001$. (D) $\mathrm{Quantification}$ of $\mathrm{tBid}$ was normalized to GAPDH. Significant differences were detected between $\mathrm{CO}$ and $\mathrm{NC}$ groups $\left({ }^{\sharp} \mathrm{P}=0.001\right)$, between $\mathrm{CO}$ and $\mathrm{AG} / \mathrm{UnAG}$ groups $\left({ }^{*} \mathrm{P}=0.006, \mathrm{P}=0.005\right.$; respectively), by one-way ANOVA followed by Tukey's test, $\mathrm{F}=14.980, \mathrm{P}=0.001$. (E) Quantification of Bid was normalized to GAPDH. Significant differences were detected between $\mathrm{CO}$ and $\mathrm{NC}$ groups ( ${ }^{\mathrm{P}<0.001)}$, between $\mathrm{CO}$ and UnAG groups ( $\left.\mathrm{P}=0.01\right)$, by one-way ANOVA followed by Tukey's test, $\mathrm{F}=62.589$, $\mathrm{P}<0.001$. (F) Quantification of mitochondrial Bcl-2 was normalized to COX IV. Significant differences were detected between CO and NC groups $\left({ }^{\#} \mathrm{P}=0.003\right)$, by one-way ANOVA followed by Tukey's test, $\mathrm{F}=10.816, \mathrm{P}=0.003$. (G) Quantification of mitochondrial Bax was normalized to COX IV. Significant differences were detected between $\mathrm{CO}$ and NC groups ( $\left.{ }^{\sharp} \mathrm{P}<0.001\right)$, between $\mathrm{CO}$ and AG/UnAG groups ( $\left.\mathrm{P}<0.001\right)$, by one-way ANOVA followed by Tukey's test, F=40.226, $\mathrm{P}<0.001$. (H) Quantification of mitochondrial Bax/Bcl-2 ratio. Significant differences were detected between $\mathrm{CO}$ and $\mathrm{NC}$ groups $\left({ }^{\sharp} \mathrm{P}<0.001\right)$, between $\mathrm{CO}$ and AG/UnAG groups ( $\mathrm{P}=0.001)$, by one-way ANOVA followed by Tukey's test, $\mathrm{F}=27.339, \mathrm{P}<0.001$. (I) The levels of Bcl-2 mRNA in myoblasts. mRNA levels were normalized to GAPDH. No significant differences were detected between 4 groups, by one-way ANOVA followed by Tukey's test. Data are represented as mean \pm SD. The coculture combinations consisted of sham myoblasts (without CT26 cells in the insert) and without ghrelin (NC group); myoblasts with CT26 cells but without ghrelin (CO group); myoblasts with CT26 cells and acylated ghrelin (AG group); myoblasts with CT26 cells and unacylated ghrelin (UnAG group).

Activity of the PI3K/Akt/glycogen synthase kinase $3 \beta$ (GSK3 $\beta$ ) pathway in myoblasts. Western blot analysis was performed to assess PI3K/Akt/GSK3 $\beta$ pathway activity. As shown in Fig. 5A and $\mathrm{B}$, the coculture was associated with a decreased level of p-AkT protein, while Akt protein levels were unaffected. This change resulted in decreased $\mathrm{p}$-AkT protein levels and p-Akt/Akt ratios in myoblasts; once again however, both AG and UnAG ameliorated these changes. No significant difference in the levels of $p$-GSK3 $\beta$ and GSK $3 \beta$ proteins was observed in the four different groups (Fig. 5C).

Concentrations of pro-inflammatory cytokines in the coculture medium. ELISA assays were performed to assess the concentrations of TNF- $\alpha$, IL- $1 \beta$, and IFN- $\gamma$ in samples of coculture medium. As shown in Fig. 6A, the coculture increased the mean TNF- $\alpha$ concentration in medium by $\sim 11$-fold, and both AG and UnAG ameliorated this affect. However, there was no statistically significant difference in the IL-1 $\beta$ and IFN- $\gamma$ concentrations in samples of culture medium from the four different groups (Fig. 6B and C).

\section{Discussion}

Cancer cachexia is a life-threatening syndrome associated with myofiber damage. Tumor factors can induce myoblast apoptosis, and thereby impair muscle regeneration. Ghrelin is a multifunctional hormone with an anti-apoptosis effect $(19,20)$. In the present study, we demonstrated that either AG or UnAG could ameliorate the increase in myoblast apoptosis caused by coculture, indicating that AG and UnAG could maintain the regeneration capability of muscle tissue, and thereby inhibit muscle atrophy.

A mitochondrial-centered control pathway is the most common mechanism of apoptosis (13), and changes in $\Delta \psi_{\mathrm{m}}$ can serve as markers of mitochondrial function (27). In this scenario, apoptotic signals converge at mitochondrial 
A

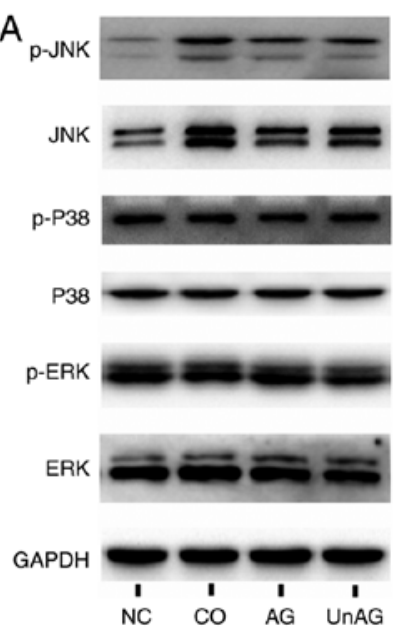

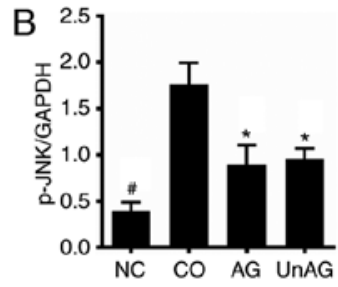

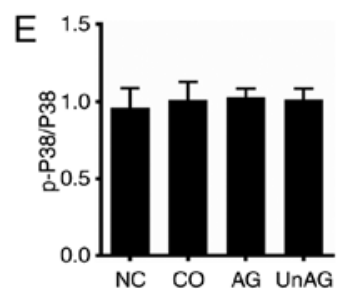

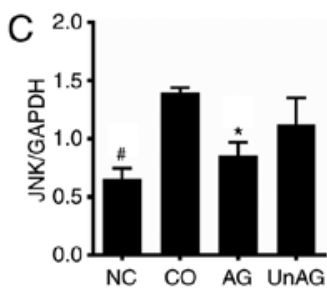
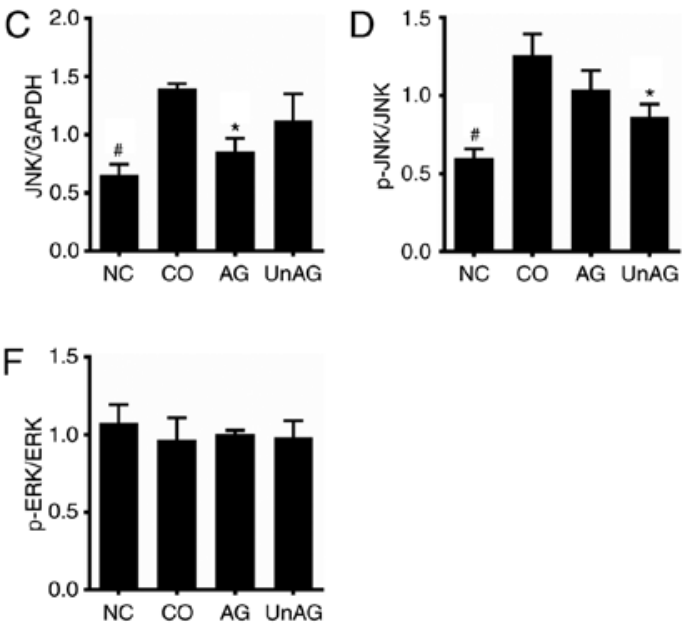

Figure 4. Regulation of the MAPK pathway by coculture and AG/UnAG administration in myoblasts. (A) Western blotting of protein levels. (B) Quantification of p-JNK was normalized to GAPDH. Significant differences were detected between $\mathrm{CO}$ and $\mathrm{NC}$ groups $\left({ }^{\sharp} \mathrm{P}<0.001\right)$, between $\mathrm{CO}$ and $\mathrm{AG} / \mathrm{UnAG}$ groups ( $\mathrm{P}=0.001, \mathrm{P}=0.002$; respectively), by one-way ANOVA followed by Tukey's test, $\mathrm{F}=32.370, \mathrm{P}<0.001$. (C) Quantification of JNK was normalized to GAPDH. Significant differences were detected between $\mathrm{CO}$ and NC groups $\left({ }^{*} \mathrm{P}=0.001\right)$, between $\mathrm{CO}$ and AG groups $\left({ }^{*} \mathrm{P}=0.005\right)$, by one-way ANOVA followed by Tukey's test, $\mathrm{F}=17.031, \mathrm{P}=0.001$. (D) Quantification of $\mathrm{p}-\mathrm{JNK} / \mathrm{JNK}$ ratio. Significant differences were detected between $\mathrm{CO}$ and NC groups ( $\left.{ }^{*} \mathrm{P}=0.001\right)$, between $\mathrm{CO}$ and UnAG groups ( $\mathrm{P}=0.013$ ), by one-way ANOVA followed by Tukey's test, $\mathrm{F}=16.480, \mathrm{P}=0.001$. (E) Quantification of $\mathrm{p}-\mathrm{P} 38$ was normalized to $\mathrm{P} 38$. No significant differences were detected between 4 groups, by one-way ANOVA followed by Tukey's test. (F) Quantification of p-ERK was normalized to ERK No significant differences were detected between 4 groups, by one-way ANOVA followed by Tukey's test. Data are represented as mean \pm SD. The coculture combinations consisted of sham myoblasts (without CT26 cells in the insert) and without ghrelin (NC group); myoblasts with CT26 cells but without ghrelin (CO group); myoblasts with CT26 cells and acylated ghrelin (AG group); myoblasts with CT26 cells and unacylated ghrelin (UnAG group).

A

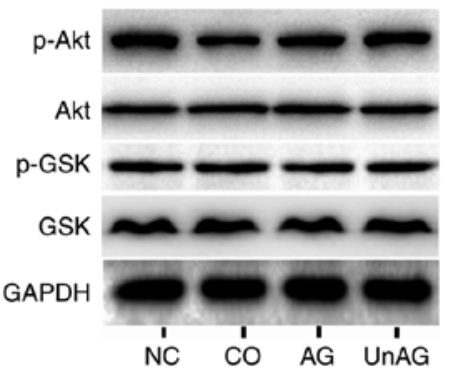

B

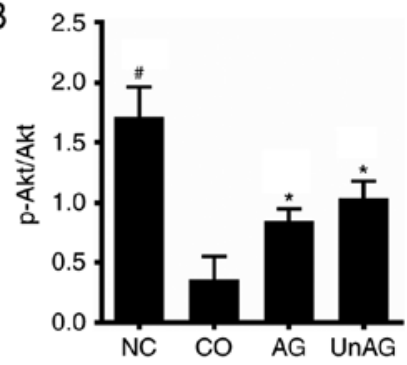

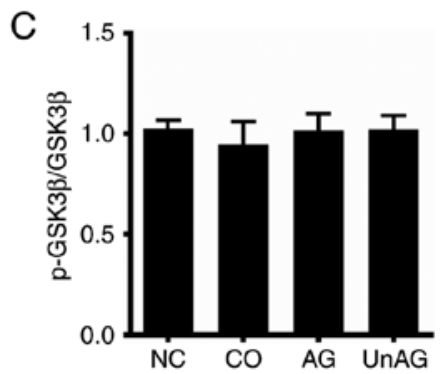

Figure 5. Regulation of the Akt/GSK3 $\beta$ pathway by coculture and AG/UnAG administration in myoblasts. (A) Western blotting of protein levels. (B) Quantification of p-Akt was normalized to Akt. Significant differences were detected between CO and NC groups ( $\left.{ }^{*} \mathrm{P}<0.001\right)$, between CO and AG/UnAG groups ( $\mathrm{P}=0.04, \mathrm{P}=0.007$; respectively), by one-way ANOVA followed by Tukey's test, $\mathrm{F}=29.901, \mathrm{P}<0.001$. (C) Quantification of $\mathrm{p}$-GSK3 $\beta$ was normalized to GSK $3 \beta$. No significant differences were detected between 4 groups, by one-way ANOVA followed by Tukey's test. Data are represented as mean \pm SD. The coculture combinations consisted of sham myoblasts (without CT26 cells in the insert) and without ghrelin (NC group); myoblasts with CT26 cells but without ghrelin (CO group); myoblasts with CT26 cells and acylated ghrelin (AG group); myoblasts with CT26 cells and unacylated ghrelin (UnAG group).

membranes, where they cause MOMP and the loss of $\Delta \psi_{\mathrm{m}}$, which lead to the release of toxic proteins (such as CYTC) from the mitochondria into cytosol (28). Next, the cytosolic CYTC works in conjunction with APAF1 to form an apoptosome, which triggers the caspase-3/PARP proteolytic cascade; which in turn, activates the downstream pathway to induce apoptotic cellular dismantling and clearance $(29,30)$. Our results showed that both $\mathrm{AG}$ and $\mathrm{UnAG}$ prevented the loss of $\Delta \psi_{\mathrm{m}}$ induced by the coculture. Moreover, AG and UnAG also inhibited the activation of caspase-3 and PARP. These findings indicate that $\mathrm{AG}$ and $\mathrm{UnAG}$ protect myoblasts from apoptosis by inhibiting coculture-induced mitochondrial damage.

The Bcl-2 family of proteins plays a critical role in regulating mitochondrial integrity. In healthy cells, Bax proteins reside mainly in the cytosol $(31,32)$. Apoptotic stimuli activate Bax proteins and target them to the MOM. The Bax protein molecules then form dimers and larger oligomers with each other and create pores directly in the MOM that result in cytochrome $c$ release and apoptotic cell death (33). The Bcl-2 protein is exclusively membrane-bound and attaches to various organelles, including mitochondria. Bcl- 2 can bind to the active form of Bax and inhibit its activity to protect mitochondrial integrity. The Bax/Bcl-2 ratio in mitochondria determines how mitochondria response to apoptotic stimuli (34). A previous study demonstrated that tumor factors increase the Bax/Bcl-2 protein ratio in skeletal muscle tissue (6). Similar to that finding, our results showed that the coculture increased the levels of Bax protein and decreased the levels of $\mathrm{Bcl}-2$ protein in mitochondria. These changes increased the $\mathrm{Bax} / \mathrm{Bcl}-2$ ratio in mitochondria and induced apoptosis. The coculture also decreased the Bax protein levels in the cytosol, which indicated that Bax became targeted to mitochondria during the coculture. We found that both AG and UnAG ameliorated these events to protect mitochondria, which is consistent with results from previous 

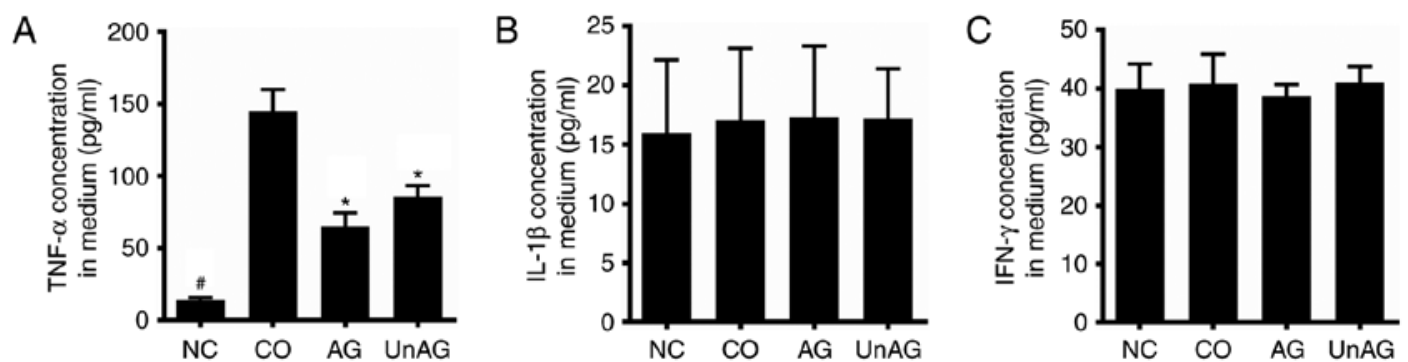

Figure 6. Concentrations of pro-inflammatory factors in the cell culture medium. (A) ELISA for TNF- $\alpha$ concentrations in coculture medium. Significant differences were detected between $\mathrm{CO}$ and $\mathrm{NC}$ groups $\left({ }^{*} \mathrm{P}<0.001\right)$, between $\mathrm{CO}$ and $\mathrm{AG} / \mathrm{UnAG}$ groups (" $\left.\mathrm{P}<0.001\right)$, by one-way ANOVA followed by Tukey's test, $\mathrm{F}=94.278, \mathrm{P}<0.001$. (B) ELISA for IL-1 $\beta$ concentrations in coculture medium. No significant differences were detected among the 6 groups, by one-way ANOVA followed by Tukey's test. (C) ELISA for IFN- $\gamma$ concentrations in coculture medium. No significant differences were detected among the 6 groups, by one-way ANOVA followed by Tukey's test. Data are represented as mean \pm SD. The coculture combinations consisted of sham myoblasts (without CT26 cells in the insert) and without ghrelin (NC group); myoblasts with CT26 cells but without ghrelin (CO group); myoblasts with CT26 cells and acylated ghrelin (AG group); myoblasts with CT26 cells and unacylated ghrelin (UnAG group).

studies $(23,24,26)$. Bcl-2 has a long biological half-life, and its expression does not significantly change during apoptosis (35). Consistent with that finding, we did not detect any significant differences in the levels of Bcl-2 mRNA and cytoplasmic protein in the four different groups.

Bax primarily resides in the cytosol and must be activated by tBid to help target it towards the MOM (36). Consistent with that finding, our results showed that the coculture increased the levels of tBid and decreased the levels of Bid in the cytosol, suggesting that the coculture had activated Bid to induce the activation of Bax. Both AG and UnAG inhibited the activation of Bid to prevent apoptosis.

Previous studies demonstrated that apoptotic stimuli activate MAPK proteins to regulate the activity of Bcl-2 family proteins $(16,34,37,38)$. Both JNK and P38 can phosphorylate Bcl-2 to inhibit its anti-apoptotic activity. JNK can also activate Bid via activation of caspase- 8 . AG has been reported to inhibit both JNK and P38 activity, and thereby regulate the activity of Bcl-2 family proteins (24). Moreover, AG and UnAG can also activate ERK to inhibit apoptosis $(22,23)$. In the present study, we found that the coculture increased the levels of p-JNK and JNK proteins, and also the p-JNK/JNK ratio in myoblasts, which indicated the activation of JNK. We also found that both AG and UnAG suppressed these increases. These data suggest that $\mathrm{AG}$ and UnAG can ameliorate the increase in JNK activity caused by coculture. However, we did not detect any difference in the levels of p-P38, P38, p-ERK and ERK proteins in the four different groups. Taken together, our results indicate that AG and UnAG suppressed JNK activity, and thereby attenuated the coculture-induced apoptosis of myoblasts.

Activated Akt promotes the survival of various cell types and prevents apoptosis induced by various stimuli (39-41). Both AG and UnAG inhibit apoptosis via activation of Akt $(22,23)$. Consistent with those findings, our results showed that $\mathrm{AG}$ and UnAG ameliorated the decreased Akt activity in myoblasts (as shown by a decreased p-Akt/Akt ratio) induced by the coculture. Akt has been reported to inhibit apoptosis via phosphorylation of its downstream target, $\operatorname{GSK} 3 \beta(23,40)$. Phosphorylation of GSK3 $\beta$ inhibits its pro-apoptosis activity. However, in the present study, the levels of p-GSK3 $\beta$ and GSK3 $\beta$ were not affected by either the coculture or AG/UnAG administration. These discrepancies are likely related to differences in the strain of the cells, the apoptotic stimuli, the dose of ghrelin administered, and the duration of exposure. Akt also inhibits the cleavage of Bid, which suppresses the production of tBid to prevent the activation of Bax. Thus, Akt prevents cell apoptosis by inhibiting Bax activity (39). Akt may act through this mechanism to inhibit coculture-induced apoptosis in myoblasts. Taken together, these data indicate that both AG and UnAG suppressed coculture-induced myoblast apoptosis via activation of Akt.

Increased levels of serum pro-inflammatory cytokines secreted by either immune cells or tumors are commonly seen in cancer cachexic animals $(2,42,43)$. Several pro-inflammatory cytokines are known to stimulate cell apoptosis via activation of JNK and inhibition of Akt $(38,44)$. In the present study, we found that coculture increased the TNF- $\alpha$ concentrations in samples of culture medium. Moreover, both AG and UnAG attenuated those increases, indicating that $A G$ and UnAG inhibited TNF- $\alpha$ secretion, and thus impaired TNF- $\alpha$-induced apoptosis in myoblasts.

Cancer cachexia causes $\sim 20 \%$ of all cancer-related deaths (2), and its pathogenesis is not completely understood. Although some tumor-bearing animal models have been developed to study cancer cachexia $(45,46)$, to the best of our knowledge, cell coculture models have never been used to simulate cancer cachexic muscle apoptosis. Our cell coculture model uses a Transwell system to grow two types of cells in the same culture medium, and allows intercellular communications to occur via cellular secretions. This model has been used to investigate cell-cell interactions between multiple cell types, such as between adipocytes and skeletal muscle fibers (47), and osteoblasts and mesenchymal stromal cells (48). In the present study, coculture of $\mathrm{C} 2 \mathrm{C} 12$ myoblasts with CT26 colon carcinoma cells increased the TNF- $\alpha$ concentrations in samples of culture medium and induced apoptosis in myoblasts, indicating that these two types of cells had interacted with each other via secreted factors. The muscle apoptosis associated with cancer cachexia results from tumor-host interactions mediated by pro-inflammatory cytokines. Moreover, increased TNF- $\alpha$ concentrations are often detected in tumor-bearing animals and cancer cachexic patients $(2,42,43)$. Such findings suggest that our coculture model can, at least in part, simulate cancer-induced muscle 
satellite cell apoptosis in vitro. Since many other tumor factors are also involved in cancer cachexia, additional studies are needed to investigate whether these factors are involved in our coculture model.

To the best of our knowledge, we demonstrated for the first time that AG and UnAG inhibit cancer-induced apoptosis in myoblasts. Previous studies have shown that both AG and UnAG can directly act on skeletal muscles, which contain numerous high-affinity binding sites $(21,49,50)$. However, the identity of the $\mathrm{AG} / \mathrm{UnAG}$ receptor remains unknown, and requires further investigation.

In conclusion, cancer cachexia is a devastating syndrome for cancer patients, and elucidating the mechanisms involved in such cachexia should enable the development of new treatment agents that can improve patient survival and quality of life. We demonstrated that coculture of $\mathrm{C} 2 \mathrm{C} 12$ myoblasts with CT26 colon carcinoma cells increased the TNF- $\alpha$ concentrations in culture medium. Additionally, the coculture activated JNK and suppressed Akt activity to regulate the activity of Bcl-2 family proteins and impair mitochondrial integrity. This impairment led to myoblast apoptosis. AG and UnAG inhibited all of these effects and protected cocultured myoblasts against apoptosis. Based on these results, we proposed that our cell coculture model can simulate cancer-induced myoblast apoptosis, and represents a new approach for investigating cancer cachexic myoblast apoptosis in vitro. We also speculate that $\mathrm{AG}$ and $\mathrm{UnAG}$ can maintain the regeneration capability of muscle tissue, and thereby attenuate cancer-induced muscle atrophy by inhibiting myoblast apoptosis. Thus, the findings described in the present study may contribute to development of an AG/UnAG-based treatment for cancer cachexia.

\section{Acknowledgements}

The present study was supported by the National Natural Science Foundation of China (no. 81272465).

\section{References}

1. Fearon K, Strasser F, Anker SD, Bosaeus I, Bruera E, Fainsinger RL, Jatoi A, Loprinzi C, MacDonald N, Mantovani G, et al: Definition and classification of cancer cachexia: An international consensus. Lancet Oncol 12: 489-495, 2011.

2. Donohoe CL, Ryan AM and Reynolds JV: Cancer cachexia: Mechanisms and clinical implications. Gastroenterol Res Pract 2011: 601434, 2011.

3. Glass DJ: Signaling pathways perturbing muscle mass. Curr Opin Clin Nutr Metab Care 13: 225-229, 2010.

4. Schiaffino S, Dyar KA, Ciciliot S, Blaauw B and Sandri M: Mechanisms regulating skeletal muscle growth and atrophy. FEBS J 280: 4294-4314, 2013.

5. Busquets S, Figueras MT, Fuster G, Almendro V, Moore-Carrasco R, Ametller E, Argilés JM and LópezSoriano FJ: Anticachectic effects of formoterol: A drug for potential treatment of muscle wasting. Cancer Res 64: 6725-6731, 2004.

6. Ishiko O, Sumi T, Yoshida H, Hyun Y and Ogita S: Expression of apoptosis regulatory proteins in the skeletal muscle of tumor-bearing rabbits compared with diet-restricted rabbits. Int J Mol Med 8: 279-283, 2001.

7. Belizário JE, Lorite MJ and Tisdale MJ: Cleavage of caspases-1, $-3,-6,-8$ and -9 substrates by proteases in skeletal muscles from mice undergoing cancer cachexia. Br J Cancer 84: 1135-1140, 2001.
8. He WA, Berardi E, Cardillo VM, Acharyya S, Aulino P, Thomas-Ahner J, Wang J, Bloomston M, Muscarella P, Nau P, et al: NF- $\mathrm{BB}$-mediated $\operatorname{Pax} 7$ dysregulation in the muscle microenvironment promotes cancer cachexia. J Clin Invest 123: 4821-4835, 2013.

9. Bossola M, Marzetti E, Rosa F and Pacelli F: Skeletal muscle regeneration in cancer cachexia. Clin Exp Pharmacol Physiol 43: $522-527,2016$.

10. Chargé SB and Rudnicki MA: Cellular and molecular regulation of muscle regeneration. Physiol Rev 84: 209-238, 2004.

11. Talbert EE and Guttridge DC: Impaired regeneration: A role for the muscle microenvironment in cancer cachexia. Semin Cell Dev Biol 54: 82-91, 2016.

12. He WA, Calore F, Londhe P, Canella A, Guttridge DC and Croce CM: Microvesicles containing miRNAs promote muscle cell death in cancer cachexia via TLR7. Proc Natl Acad Sci USA 111: 4525-4529, 2014.

13. Hotchkiss RS, Strasser A, McDunn JE and Swanson PE: Cell death. N Engl J Med 361: 1570-1583, 2009.

14. Green DR: Apoptotic pathways: Ten minutes to dead. Cell 121: 671-674, 2005.

15. Moldoveanu T, Follis AV, Kriwacki RW and Green DR: Many players in BCL-2 family affairs. Trends Biochem Sci 39: 101-111, 2014.

16. Deng Y, Ren X, Yang L, Lin Y and Wu X: A JNK-dependent pathway is required for TNFalpha-induced apoptosis. Cell 115: 61-70, 2003.

17. Gnanapavan S, Kola B, Bustin SA, Morris DG, McGee P, Fairclough P, Bhattacharya S, Carpenter R, Grossman AB and Korbonits M: The tissue distribution of the mRNA of ghrelin and subtypes of its receptor, GHS-R, in humans. J Clin Endocrinol Metab 87: 2988, 2002

18. Molfino A, Gioia G and Muscaritoli M: The hunger hormone ghrelin in cachexia. Expert Opin Biol Ther 13: 465-468, 2013.

19. Chopin L, Walpole C, Seim I, Cunningham P, Murray R, Whiteside E, Josh P and Herington A: Ghrelin and cancer. Mol Cell Endocrinol 340: 65-69, 2011.

20. Guillory B, Splenser A and Garcia J: The role of ghrelin in anorexia-cachexia syndromes. Vitam Horm 92: 61-106, 2013.

21. Filigheddu N, Gnocchi VF, Coscia M, Cappelli M, Porporato PE, Taulli R, Traini S, Baldanzi G, Chianale F, Cutrupi S, et al: Ghrelin and des-acyl ghrelin promote differentiation and fusion of $\mathrm{C} 2 \mathrm{C} 12$ skeletal muscle cells. Mol Biol Cell 18: 986-994, 2007.

22. Baldanzi G, Filigheddu N, Cutrupi S, Catapano F, Bonissoni S, Fubini A, Malan D, Baj G, Granata R, Broglio F, et al: Ghrelin and des-acyl ghrelin inhibit cell death in cardiomyocytes and endothelial cells through ERK1/2 and PI 3-kinase/AKT. J Cell Biol 159: 1029-1037, 2002.

23. Chung H, Seo S, Moon M and Park S: Phosphatidylinositol3-kinase/Akt/glycogen synthase kinase-3 beta and ERK1/2 pathways mediate protective effects of acylated and unacylated ghrelin against oxygen-glucose deprivation-induced apoptosis in primary rat cortical neuronal cells. J Endocrinol 198: 511-521, 2008.

24. Mao Y, Wang J, Yu F, Li Z, Li H, Guo C and Fan X: Ghrelin protects against palmitic acid or lipopolysaccharide-induced hepatocyte apoptosis through inhibition of MAPKs/iNOS and restoration of Akt/eNOS pathways. Biomed Pharmacother 84: 305-313, 2016.

25. Yu J, Xu H, Shen X and Jiang H: Ghrelin protects MES23.5 cells against rotenone via inhibiting mitochondrial dysfunction and apoptosis. Neuropeptides 56: 69-74, 2016.

26. Zhang Q, Huang WD, Lv XY and Yang YM: Ghrelin protects $\mathrm{H} 9 \mathrm{c} 2$ cells from hydrogen peroxide-induced apoptosis through NF- $\kappa \mathrm{B}$ and mitochondria-mediated signaling. Eur J Pharmacol 654: 142-149, 2011.

27. Galluzzi L, Vitale I, Abrams JM, Alnemri ES, Baehrecke EH, Blagosklonny MV, Dawson TM, Dawson VL, El-Deiry WS, Fulda S, et al: Molecular definitions of cell death subroutines: Recommendations of the nomenclature committee on cell death 2012. Cell Death Differ 19: 107-120, 2012.

28. Kroemer G, Galluzzi L and Brenner C: Mitochondrial membrane permeabilization in cell death. Physiol Rev 87: 99-163, 2007.

29. Li P, Nijhawan D, Budihardjo I, Srinivasula SM, Ahmad M, Alnemri ES and Wang X: Cytochrome $\mathrm{c}$ and dATP-dependent formation of Apaf-1/caspase-9 complex initiates an apoptotic protease cascade. Cell 91: 479-489, 1997.

30. Zou H, Henzel WJ, Liu X, Lutschg A and Wang X: Apaf-1, a human protein homologous to C. elegans CED-4, participates in cytochrome c-dependent activation of caspase-3. Cell 90: 405-413, 1997. 
31. Hsu YT, Wolter KG and Youle RJ: Cytosol-to-membrane redistribution of Bax and Bcl- $\mathrm{X}_{\mathrm{L}}$ during apoptosis. Proc Natl Acad Sci USA 94: 3668-3672, 1997.

32. Wolter KG, Hsu YT, Smith CL, Nechushtan A, Xi XG and Youle RJ: Movement of Bax from the cytosol to mitochondria during apoptosis. J Cell Biol 139: 1281-1292, 1997.

33. Gross A, Jockel J, Wei MC and Korsmeyer SJ: Enforced dimerization of BAX results in its translocation, mitochondrial dysfunction and apoptosis. EMBO J 17: 3878-3885, 1998.

34. Yamamoto K, Ichijo $\mathrm{H}$ and Korsmeyer SJ: BCL-2 is phosphorylated and inactivated by an ASK1/Jun N-terminal protein kinase pathway normally activated at $\mathrm{G}_{2} / \mathrm{M}$. Mol Cell Biol 19: 8469-8478, 1999.

35. Willimott S and Wagner SD: Post-transcriptional and post-translational regulation of Bcl2. Biochem Soc Trans 38: 1571-1575, 2010.

36. Walensky LD: Direct BAKtivation. Nat Struct Mol Biol 20: 536-538, 2013.

37. De Chiara G, Marcocci ME, Torcia M, Lucibello M, Rosini P, Bonini P, Higashimoto Y, Damonte G, Armirotti A, Amodei S, et al: Bcl-2 Phosphorylation by p38 MAPK: Identification of target sites and biologic consequences. J Biol Chem 281: 21353-21361, 2006.

38. Kyriakis JM and Avruch J: Mammalian MAP004B signal transduction pathways activated by stress and inflammation: A 10-year update. Physiol Rev 92: 689-737, 2012.

39. Yamaguchi $\mathrm{H}$ and Wang HG: The protein kinase PKB/Akt regulates cell survival and apoptosis by inhibiting Bax conformational change. Oncogene 20: 7779-7786, 2001.

40. Zhu Z, Dai J, Liao Y and Wang T: Sox9 protects against human lung fibroblast cell apoptosis induced by LPS through activation of the AKT/GSK3 $\beta$ pathway. Biochemistry 82: 606-612, 2017.

41. Li S, Chen JW, Xie X, Tian J, Deng C, Wang J, Gan HN and Li F: Autophagy inhibitor regulates apoptosis and proliferation of synovial fibroblasts through the inhibition of PI3K/AKT pathway in collagen-induced arthritis rat model. Am J Transl Res 9: 2065-2076, 2017.

42. Fearon KC, Glass DJ and Guttridge DC: Cancer cachexia: Mediators, signaling, and metabolic pathways. Cell Metab 16 $153-166,2012$.
43. Argiles JM, Busquets S, Stemmler B and López-Soriano FJ: Cancer cachexia: Understanding the molecular basis. Nat Rev Cancer 14: 754-762, 2014

44. Granata R, Settanni F, Biancone L, Trovato L, Nano R, Bertuzzi F, Destefanis S, Annunziata M, Martinetti M, Catapano F, et al: Acylated and unacylated ghrelin promote proliferation and inhibit apoptosis of pancreatic beta-cells and human islets: Involvement of 3',5'-cyclic adenosine monophosphate/protein kinase a, extracellular signal-regulated kinase $1 / 2$, and phosphatidyl inositol 3-Kinase/Akt signaling. Endocrinology 148: 512-529, 2007.

45. Tsubouchi H, Yanagi S, Miura A, Matsumoto N, Kangawa K and Nakazato M: Ghrelin relieves cancer cachexia associated with the development of lung adenocarcinoma in mice. Eur J Pharmacol 743: 1-10, 2014.

46. Penna F, Costamagna D, Pin F, Camperi A, Fanzani A, Chiarpotto EM, Cavallini G, Bonelli G, Baccino FM and Costelli P: Autophagic degradation contributes to muscle wasting in cancer cachexia. Am J Pathol 182: 1367-1378, 2013.

47. Wohlers LM, Powers BL, Chin ER and Spangenburg EE: Using a novel coculture model to dissect the role of intramuscular lipid load on skeletal muscle insulin responsiveness under reduced estrogen conditions. Am J Physiol Endocrinol Metab 304: E1199-E1212, 2013.

48. Voss JO, Loebel C, Bara JJ, Fussinger MA, Duttenhoefer F, Alini M and Stoddart MJ: Effect of short-term stimulation with interleukin-1 $\beta$ and differentiation medium on human mesenchymal stromal cell paracrine activity in coculture with osteoblasts. Biomed Res Int 2015: 714230, 2015.

49. Sheriff S, Kadeer N, Joshi R, Friend LA, James JH and Balasubramaniam A: Des-acyl ghrelin exhibits pro-anabolic and anti-catabolic effects on $\mathrm{C} 2 \mathrm{C} 12$ myotubes exposed to cytokines and reduces burn-induced muscle proteolysis in rats. Mol Cell Endocrinol 351: 286-295, 2012.

50. Porporato PE, Filigheddu N, Reano S, Ferrara M, Angelino E, Gnocchi VF, Prodam F, Ronchi G, Fagoonee S, Fornaro M, et al: Acylated and unacylated ghrelin impair skeletal muscle atrophy in mice. J Clin Invest 123: 611-622, 2013. 